\title{
Formal thought disorder in people at ultra-high risk of psychosis
}

Arsime Demjaha, Sara Weinstein, Daniel Stahl, Fern Day, Lucia Valmaggia, Grazia Rutigliano, Andrea De Micheli, Paolo Fusar-Poli and Philip McGuire

\section{Background}

Formal thought disorder is a cardinal feature of psychosis. However, the extent to which formal thought disorder is evident in ultra-high-risk individuals and whether it is linked to the progression to psychosis remains unclear.

\section{Aims}

Examine the severity of formal thought disorder in ultrahigh-risk participants and its association with future psychosis.

\section{Method}

The Thought and Language Index (TLI) was used to assess 24 ultra-high-risk participants, 16 people with first-episode psychosis and 13 healthy controls. Ultra-high-risk individuals were followed up for a mean duration of 7 years $(s . d .=1.5)$ to determine the relationship between formal thought disorder at baseline and transition to psychosis.

\section{Results}

TLI scores were significantly greater in the ultra-high-risk group compared with the healthy control group (effect size $(E S)=1.2$ )
Formal thought disorder is a cardinal symptom of psychotic disorders. ${ }^{1}$ Although initially considered as part of the positive symptom construct, studies have confirmed that it constitutes a separate symptom dimension. ${ }^{2,3}$ It has been shown that formal thought disorder is associated with most severe forms of illness, ${ }^{4,5}$ persists after the resolution of positive symptoms, ${ }^{6}$ and leads to poor outcome. ${ }^{7}$ Furthermore, neuroimaging studies have confirmed its distinct structural and functional correlates, ${ }^{8,9}$ which differ for its negative ${ }^{10}$ and positive components, ${ }^{8}$ and there is evidence that genetic vulnerability to psychosis is strongly associated with formal thought disorder. ${ }^{11}$ Although formal thought disorder is clearly an important feature of psychotic disorders, the extent to which it is evident in people who are at ultra-high risk for psychosis is unclear. The main instruments that are used to assess psychopathology in ultra-high-risk individuals, the Comprehensive Assessment of the At-Risk Mental State (CAARMS), ${ }^{12}$ the Structured Interview for Psychosis-Risk Syndromes (SIPS) and the Schizophrenia Prediction Instrument - Adult version (SPI-A) ${ }^{13}$ include items that correspond to features of formal thought disorder with evidence that ultra-high-risk individuals score positively on these items, ${ }^{14,15}$ however, these instruments are not specific in assessing and quantifying the less obvious or vague form of thought disorder that is characteristic of prodromal phase of illness. We documented that transition to psychosis was associated to CAARMS ratings for disorganised speech ${ }^{4}$ and three other studies have reported a similar association with disorganised communication. ${ }^{16-18}$ Hartmann and colleagues $^{19}$ speculated that formal thought disorder may be a robust predictor of later psychosis, which is consistent with the child and adolescents studies that used specific formal thought disorder assessment tools and observed that thought disorder in childhood or adolescence predicted psychosis in adulthood. ${ }^{11,14,15,20}$ To date, no studies have examined formal thought disorder in ultrahigh-risk individuals by using instruments that have been specifically designed to evaluate formal thought disorder. The Thought but lower than in people with first-episode psychosis ( $E S=0.8$ ). Total and negative TLI scores were higher in ultra-high-risk individuals who developed psychosis, but this was not significant. Combining negative TLI scores with attenuated psychotic symptoms and basic symptoms predicted transition to psychosis $(P=0.04$; $E S=1.04)$

\section{Conclusions}

TLI is beneficial in evaluating formal thought disorder in ultrahigh-risk participants, and complements existing instruments for the evaluation of psychopathology in this group.

\section{Declaration of interests}

None.

\section{Copyright and usage}

(c) The Royal College of Psychiatrists 2017. This is an open access article distributed under the terms of the Creative Commons Non-Commercial, No Derivatives (CC BY-NC-ND) license.

and Language Index (TLI) is one such instrument, sensitive to subtle language anomalies and has previously been successfully applied in people with schizophrenia, non-clinical individuals with psychotic symptoms and healthy volunteers. ${ }^{8,21,22}$ Our aim was to use the TLI to determine whether formal thought disorder is a feature of the ultra-high-risk state, and to compare it with thought disorder in people with a psychotic disorder. A further objective was to investigate whether the severity of formal thought disorder in ultra-high-risk participants at presentation is related to transition to psychosis. In line with recent guidance on early detection measures, ${ }^{14}$ we also assessed the effect of combining TLI scores with baseline measures of attenuated psychotic symptoms and six basic symptoms that are incorporated within CAARMS. The following hypotheses were tested:

(a) Formal thought disorder is evident in the ultra-high-risk state.

(b) Formal thought disorder is qualitatively similar to, but less severe, than formal thought disorder in established psychosis.

(c) Formal thought disorder at presentation in ultra-high-risk individuals is associated with an increased risk of subsequent transition to psychosis.

\section{Method}

\section{Participants}

Ultra-high-risk individuals aged 16-35 years were recruited through Outreach and Support in South London (OASIS), a clinical service for people with an ultra-high risk in South London. ${ }^{23}$ OASIS has an ongoing programme of liaison with local health and non-health agencies who may encounter people with prodromal symptoms suggestive of an 'ultra-high risk'. The information about early recognition signs and inclusion criteria 
was disseminated through regular meetings, presentations and distribution of leaflets. Patients were referred from general practitioner surgeries, schools and colleges, social and faith groups, or adolescent and adult mental health services or have initiated contact themselves. Referred individuals were contacted by telephone for an initial screening and then an assessment with OASIS was offered. Individuals who met ultra-high-risk criteria were seen at regular intervals over the next 2 years with close clinical monitoring for signs of frank psychosis.

Transition to psychosis was defined as the onset of frank psychotic symptoms, that is, symptoms at a severity greater than that corresponding to attenuated psychotic symptoms in the CAARMS, which did not resolve within 1 week. (Severity Scale score of 6 on Disorders of Thought Content subscale, 5 or 6 on Perceptual Abnormalities subscale and/or 6 on Disorganised Speech subscales of the CAARMS).

People who presented with their first episode of psychosis were recruited from the South London and Maudsley NHS Trust. Healthy volunteers with no previous or current history of psychiatric illness (as assessed by the Structured Clinical Interview for DSM-IV Axis I Disorders and the Structured Clinical Interview for DSM-IV Personality Disorders) and no family history of psychosis, were recruited from the same geographical area by local advertisement and by approaching the social contacts of ultra-high-risk individuals after receiving written permission. They were matched to the ultra-high-risk individuals and those with first episode psychosis for age and gender.

Exclusion criteria for all participants were a history of a neurological or medical disorder (other than past minor selflimiting illnesses) or head injury; illicit drug or alcohol misuse or dependence. Each participant gave written informed consent after receiving a complete description of the study.

All participants were fluent in English and appart from two, all were right-handed.

Ethical approval for the study was obtained from the Institute of Psychiatry Research Ethics Committee. All participants gave their written informed consent to participate in this study.

\section{Measurements}

Comprehensive Assessment of the At-Risk Mental State

The presence of the ultra-high risk was determined by the CAARMS. Participants met one or more ultra-high-risk criteria: (1) a recent decline in function, combined with either a family history of psychosis in a first-degree relative or the presence of schizotypal personality disorder; (2) presence of attenuated psychotic symptoms; (3) a brief psychotic episode of less than 1 week's duration that resolves without antipsychotic medication. ${ }^{24} \mathrm{Six}$ of the Huber's basic symptoms are incorporated within the CAARMS: subjective experience of cognitive change, subjective emotional disturbance, avolition, subjective complaints of impaired motor functioning, subjective complaints of impaired bodily sensations and subjective complaints of impaired autonomic functioning. ${ }^{25}$ These were used to create an aggregate score for subsequent analyses. Scores for each subscale range from 0 to 6 . Only scores on the subscale of positive symptoms are used to evaluate the ultra-high-risk criteria.

\section{Thought and Language Index}

Formal thought disorder was assessed by the TLI. ${ }^{21}$ The TLI rates eight types of speech abnormality: looseness, peculiar word usage, peculiar sentence usage, peculiar logic (positive or disorganisation formal thought disorder subscale), poverty of speech, weakening of goal (negative formal thought disorder subscale) and perseveration and distractibility (non-specific formal thought disorder subscale).
Participants were presented with a set of eight pictures from the thematic apperception $t$-test ${ }^{26}$ in a sequential order, and required to talk about each picture for $1 \mathrm{~min}$. Their speech was recorded by a voice recorder and then subsequently transcribed. For each picture, $1 \mathrm{~min}$ of free response was followed by an enquiry phase in which the participant could be asked to clarify what they meant or said if a response had been idiosyncratic or indistinct. Transcriptions of the speech samples were scored by an assessor trained in administration of TLI (S.W.), who was masked to participant status. Individual items were assigned a score ranging from 0.25 (phenomenon of questionable deviance) to 1 (phenomenon clearly deviant) according to severity. The composite score for each subscale was obtained by summing the individual scores for the items comprising within that subscale. Each of the TLI subscale's scores were also combined with the attenuated psychotic symptoms and basic symptom scores to create a composite psychopathology score, which was designed to reflect the overall severity of attenuated psychotic symptoms, basic symptoms and formal thought disorder.

Social functioning was assessed by the Global Assessment of Functioning (GAF). ${ }^{27}$ The National Adult Reading Scale $(\mathrm{NART})^{28}$ was used to assess pre-morbid intelligence quotient (IQ), and handedness was determined by the Annet Handedness Scale. $^{29}$

\section{Statistical analyses}

Statistical analysis was performed in SPSS version 22.0 (Chicago, Illinois, USA). Preliminary tests were performed to explore homogeneity of variance, regression slopes, normality and reliable measurements of covariates. The Kolmogorov-Smirnov test revealed that TLI data had non-parametric distribution. We performed the analysis of variance (ANOVA) to test group differences for total TLI scores and TLI subscale scores. Although ANOVA is considered a robust test against the normality assumption, we repeated the analyses by non-parametric statistics (Kruskal-Wallis and MannWhitney $U$-tests). When there were significant group effects, Tukey test for multiple comparisons was performed to examine differences in TLI and its subscale' scores between groups. An additional analysis of covariance was performed with IQ and educational level added as covariates because these two items were not strongly correlated $(r=0.56)$. To examine the combined effect of TLI, attenuated psychotic symptoms and basic symptom measures, we aggregated the data by summing their standardised scores in a composite variable. Finally, we conducted $t$-tests for independent samples.

\section{Results}

Complete TLI data from 53 participants were available for analysis, including data from 24 ultra-high-risk participants, 16 individuals with first-episode psychosis and 13 healthy controls. There were no between-group differences in age, gender or ethnicity. However, the groups differed on IQ and level of education, reflecting higher values in the controls than in the two clinical groups (Table 1).

\section{Severity of formal thought disorder in ultra-high risk relative to first-episode psychosis and healthy controls}

Each of the total, positive and negative TLI scores in the ultrahigh-risk group were intermediate between those in the firstepisode psychosis group and the controls (Fig. 1).

There was a significant effect of group on TLI total scores ( $F=10.1$, d.f. $=2,50, P>0.001)$, positive TLI scores $(F=3.1$, d.f. $=2$, 50, $P=0.04)$ and negative TLI scores ( $F=6.1$, d.f. $=2,50, P=0.05)$. All of these differences remained significant when the analysis was repeated by the Kruskal-Wallis test (TLI total $(P>0.001)$, positive 


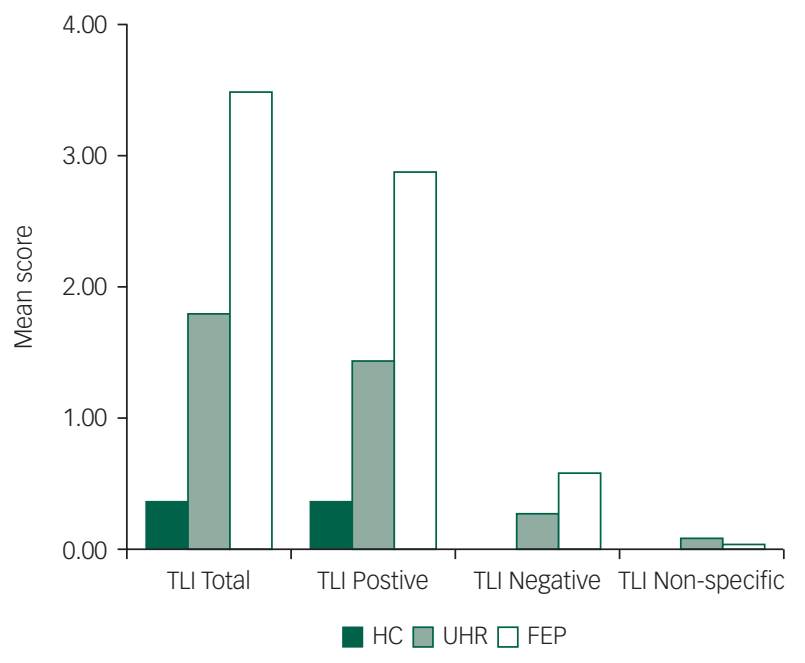

Fig. 1 Mean severity of total Thought and Language Index (TLI) and its subscale scores (positive, negative and non-specific) in the ultrahigh risk (UHR) ( $n=24)$, first-episode psychosis (FEP) $(n=16)$ and healthy control $(\mathrm{HC})(n=13)$ groups

( $P>0.001)$, TLI negative $(P=0.005)$ ). Because the groups differed in IQ and educational level, after taking account of the effects of these factors, the effects of group on the TLI total $(F=4.6, P=0.01)$ and positive $(F=3.9, P=0.03)$ scores remained significant, but there was no longer a significant difference for the TLI negative subscale. The repeat analysis taking into account IQ and educational level separately revealed similar results.

\section{Between-group comparisons}

Ultra-high risk $v$. healthy controls

Total TLI scores were significantly greater in the ultra-high-risk group compared with the healthy controls group ( $P=0.03$; corrected effect size $(E S)=1.2$ ), but the differences on the TLI subscale scores were not significant.

\section{Ultra-high risk v. first-episode psychosis}

After adjustment for multiple comparisons, total TLI and positive subscale scores were significantly greater in the first-episode psychosis group than in the ultra-high-risk group $(P=0.007$; corrected $\mathrm{ES}=0.8$ and $P=0.02$; ES $=0.7$ respectively), but there were no significant differences for the negative subscale.

\section{First-episode psychosis $v$. healthy controls}

The first-episode psychosis group scored significantly higher on total TLI $(P>0.001 ; \mathrm{ES}=1.4)$, positive $(P=0.001 ; \mathrm{ES}=1.1)$ and negative subscales $(P=0.04 ; \mathrm{ES}=0.9)$.

\section{The prevalence of formal thought disorder in the three groups}

The most prevalent TLI items were those from the positive subscale, which were evident in $75 \%$ of ultra-high-risk participants, $94 \%$ of people with first-episode psychosis and $31 \%$ of healthy controls. Negative subscale items were evident in $21 \%$ of ultra-high risk and $37 \%$ individuals with first-episode psychosis and completely absent in healthy controls. The most prevalent individual TLI items were the use of peculiar sentences (evident in $72 \%$ of ultra-high-risk participants, $82 \%$ of individuals with first-episode psychosis and 30\% of healthy controls) and use of peculiar words (25\% of ultra-high-risk participants, $37 \%$ of people with first-episode psychosis and none of healthy controls) (Fig. 2).

\section{Relationship between TLI scores and subsequent transition to psychosis}

Subsequent to assessment, the ultra-high-risk individuals were followed clinically for a mean of 7 years $($ s.d. $=1.5)$. During this period, $8(30 \%)$ had made a transition to psychosis. The median time to transition to psychosis was 24 months.

Within the ultra-high-risk sample, the severity of the total and negative TLI scores at baseline was higher in participants who subsequently made a transition to psychosis, but these differences were not statistically significant (Fig. 3). The similarity in the positive TLI scores between ultra-high-risk individuals who did or did not become psychotic suggests that the difference in the total TLI score was driven by the difference in negative TLI score (Fig. 3). The composite psychopathology score was significantly associated with later transition to psychosis when it included the TLI negative score $(P=0.04$; $E S=1.04)$, but not when it included the total or positive TLI scores. The same result was obtained when the $t$-test analyses were repeated by the Mann-Whitney $U$-test $(P=0.024)$.

\begin{tabular}{|c|c|c|c|c|}
\hline & UHR $(n=24)$ & First episode psychosis $(n=16)$ & Healthy controls $(n=13)$ & $P$ \\
\hline Age, years: mean (s.d.) & $25.17(4.82)$ & $24.47(3.70)$ & $26.54(5.21)$ & ns \\
\hline WRAT IQ, mean (s.d.) & $103.3(11.8)$ & 98.67 (14.79) & $115.62(5.19)$ & 0.001 \\
\hline Years of education, mean (s.d.) & $13.0(2.75)$ & $13.40(1.84)$ & $18.38(4.17)$ & $>0.001$ \\
\hline Male, $n(\%)$ & $15(62.5)$ & $13(81.3)$ & $8(61.5)$ & ns \\
\hline \multicolumn{5}{|l|}{ Ethnicity, $n$} \\
\hline White & 14 & 6 & 10 & ns \\
\hline Black & 5 & 7 & 2 & \\
\hline Asian & 1 & 0 & 0 & \\
\hline Mixed race & 4 & 1 & 1 & \\
\hline Medication, mg/day CPZ eqv.: mean (s.d.) & $140(42.4)$ & $150(83.7)$ & - & ns \\
\hline GAF baseline, mean (s.d.) & $59.3(14.5)$ & - & - & \\
\hline GAF follow-up, mean (s.d.) & $63.9(16.9)$ & & & \\
\hline \multicolumn{5}{|l|}{ Transition to psychosis, $n$ (\%) } \\
\hline Yes & $8(33.3)$ & & & \\
\hline No & $16(66.7)$ & & & \\
\hline
\end{tabular}




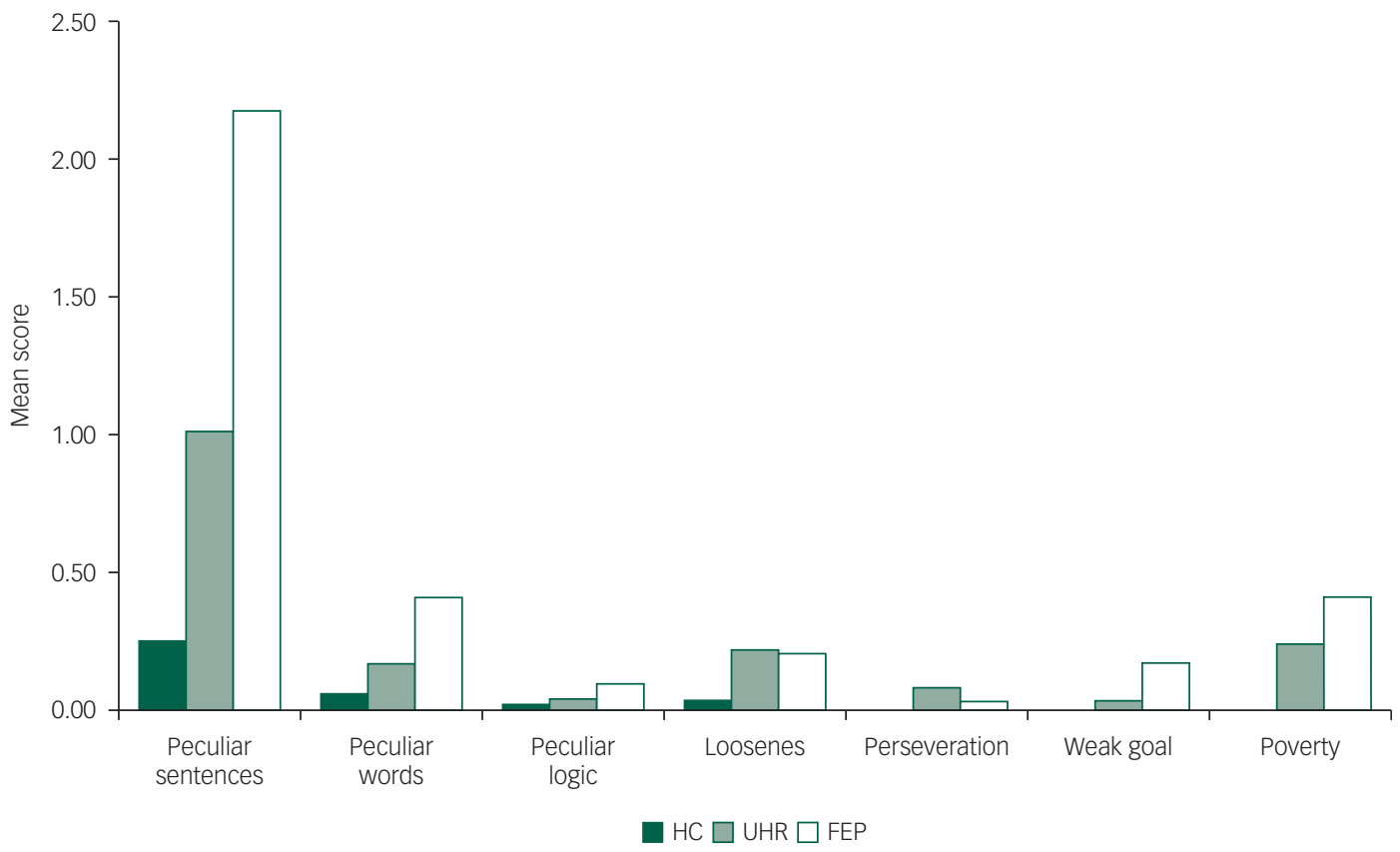

Fig. 2 Mean severity of Thought and Language Index items in the ultra-high risk (UHR) ( $n=24)$, first-episode psychosis (FEP) ( $n=16)$ and healthy control $(\mathrm{HC})(n=13)$ groups.

\section{Discussion}

\section{Formal thought disorder is evident in the ultra-high risk group}

Our first main finding was that formal thought disorder is evident in ultra-high-risk individuals. There was a clear difference between ultrahigh-risk individuals and controls in the overall severity of formal thought disorder, with an effect size of 1.2 for the total TLI score. As with abnormal beliefs and experiences, ${ }^{30}$ formal thought disorder in ultra-high-risk individuals was qualitatively similar to that in people with first-episode psychosis, but its severity was intermediate between that in people with first-episode psychosis and controls. This pattern of intermediate severity was evident for the total TLI score, as well as for the positive and negative subscale scores.

At present, the main instruments that are used to assess psychopathology in ultra-high-risk individuals are the CAARMS and SIPS. Both permit a detailed evaluation of abnormal beliefs and hallucinations, but include relatively little coverage of items relevant to formal thought disorder. The SPI-A is also frequently employed, and is used to assess basic symptoms. ${ }^{31}$ Although some of the SPI-A items relate to the abnormal expression and reception of language, these measures provide an index of the individual's subjective experience, rather than an objective assessment of the individual's speech by an assessor, as in the TLI. Consequently, most studies of psychopathology in the ultra-high-risk state to date have not assessed formal thought disorder in detail. Specialised instruments such as the TLI provide a means of addressing this issue, and can be seen as complementary to the CAARMS, SIPS and SPI-A.

Ultra-high-risk individuals scored positively on similar items to people with first-episode psychosis. However, although healthy controls also scored weakly on positive TLI items, they did not score for negative TLI items. The latter observation is consistent with previous reports that negative formal thought disorder is particularly rare in healthy volunteers, ${ }^{21,22}$ and suggests that it may be more specific for a psychotic disorder than positive formal thought disorder. Peculiar sentences and peculiar words usage were the most prevalent in all three groups, being the only type of thought disorder present in healthy controls. The finding that thought disorder is more severe in first-episode psychosis individuals compared with individuals with ultra-high risk indicates that there is further progression in the severity of formal thought disorder as individuals make the transition to frank psychosis. Similarly, the observation that positive formal thought disorder is detectable in healthy individuals, but more prevalent and severe in ultra-high risk and first-episode psychosis, lends support to the idea proposed by Liddle et al. ${ }^{21}$ 'that there might be a continuum of severity of disorganised thought in the human population'.

\section{Formal thought disorder as a predictor of clinical outcomes}

In a previous ultra-high-risk study, we found that loadings on a disorganisation/cognitive symptom dimension (that incorporated the CAARMS item 'disorganised speech') were associated with the subsequent transition to psychosis. ${ }^{3}$ Studies in other

\begin{tabular}{|c|c|c|c|c|c|}
\hline & Healthy controls $(n=13)$ & Ultra-high risk $(n=24)$ & First-episode psychosis $(n=16)$ & $F($ d.f. $=2)$ & $P$ \\
\hline TLI Total & $0.37(0.51)$ & $1.79(1.43)$ & $3.48(2.98)$ & 10.05 & $>0.001$ \\
\hline TLI Positive & $0.37(0.51)$ & $1.43(1.27)$ & $2.88(3.01)$ & 6.59 & 0.007 \\
\hline TLI Negative & $0.00(0.00)$ & $0.27(0.61)$ & $0.58(0.86)$ & 3.08 & 0.05 \\
\hline TLI Non-specific & $0.00(0.00)$ & $0.08(0.21)$ & $0.03(0.12)$ & 1.2 & 0.31 \\
\hline
\end{tabular}




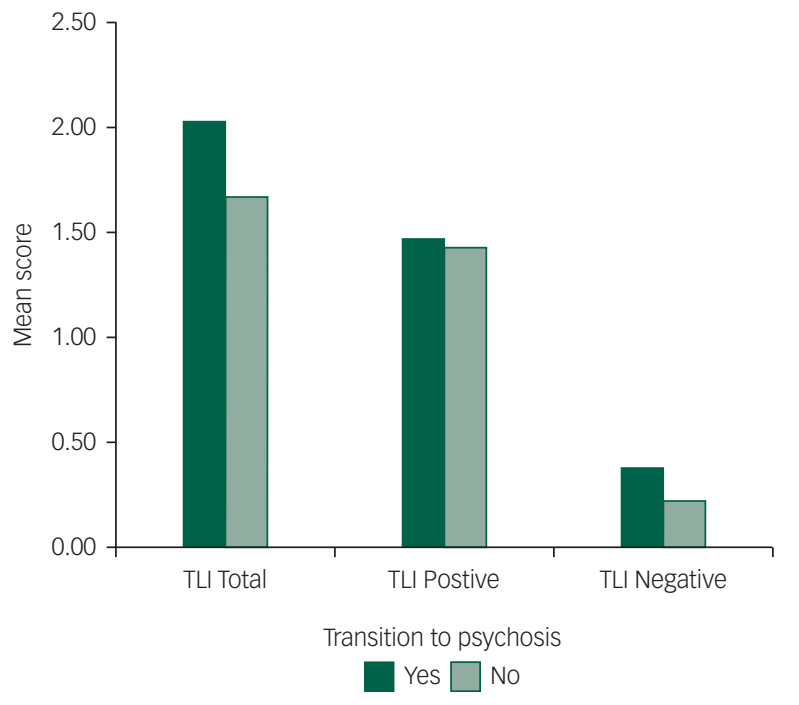

Fig. 3 Mean severity of Thought and Language Index (TLI) scores for people with and without transition to psychosis.

ultra-high-risk samples have independently reported associations between 'disorganised communication' (an item within the SIPS) and the later onset of psychosis. ${ }^{16-18}$ In addition, prospective studies in community samples of children and adolescents have observed associations between subtle thought disorder and communication disturbance with the onset of psychosis in later life. ${ }^{11,32-34}$ The potential importance of ratings of formal thought disorder in predicting the risk of later psychosis has recently been recognised in the European Psychiatric Association (EPA) guidelines on early detection, which recommends combining scores on the 'disorganised speech' item from the CAARMS or the SIPS with ratings of attenuated psychotic symptoms and basic symptoms as an index of risk of later transition. ${ }^{14}$ In the present study, we found that combining negative TLI scores with these ratings was associated with an increased incidence of transition to psychosis, in line with these guidelines.

It is noteworthy that negative formal thought disorder relates to negative symptom sub-domain, diminished expression. Its association with later transition to psychosis in the ultra-high risk is thus consistent with previous evidence linking the severity of negative symptoms more generally in the ultra-high risk and the subsequent onset of psychosis. ${ }^{3,35}$ However, contrary to our hypothesis, the severity of formal thought disorder alone, as determined by the TLI, was not associated with later transition to psychosis. This might have been an effect of our modest sample size, which meant that the number of participants in the transition subgroup $(n=8)$ may have been too small to permit the detection of a true difference. Negative formal thought disorder was not only more frequently present in ultra-high-risk participants who subsequently developed psychosis, unlike positive formal thought disorder, it was not detectable in the healthy volunteers.

\section{Limitations}

The group sizes in our study were modest, partly because the TLI is a relatively complicated and time-consuming instrument to administer. Our modest samples sizes and low average TLI negative scores in ultra-high risk and first episode psychosis samples, may have limited our statistical power to detect true differences, thus our results require replication, ideally in prospective studies of large samples.

To derive a composite psychopathology score, we used the rating of basic symptoms that are incorporated within the CAARMS.
Ideally, we would have used an instrument that is specifically designed to assess basic symptoms, such as the SPI-A. ${ }^{31}$ The use of CAARMS to assess basic symptoms may have reduced the predictive power of the composite score.

Although only a minority $(n=4)$ of the ultra-high-risk participants were taking antipsychotic medication, we cannot exclude the possibility that this may have influenced the results. However, the lower severity of formal thought disorder in the ultra-high-risk participants than in the people with psychosis cannot be attributed to an effect of antipsychotic medication, as a far greater proportion of the first-episode group were being treated with antipsychotics.

In conclusion, formal thought disorder is evident in people at ultra-high risk for psychosis and is qualitatively similar to that seen in people with psychotic disorders, but less severe. Specialised instruments developed for the assessment of formal thought disorder in people with psychosis, such as the TLI, provide a means of evaluating formal thought disorder in ultra-high-risk participants, and complement existing instruments for the evaluation of psychopathology in this group. Prospective studies in large samples are required to confirm whether assessing the severity of formal thought disorder may be useful in helping to predict whether an individual at high risk will go on to develop psychosis.

\footnotetext{
Arsime Demjaha, PhD, Department of Psychosis Studies, Biomedical Research Centre, Institute of Psychiatry, Psychology and Neuroscience, King's College London, UK; Sara Weinstein, PhD, Boeing Vancouver Labs, Vancuver, British Columbia, Canada; Daniel Stahl, PhD, Department of Biostatistics, Institute of Psychiatry, Psychology and Neuroscience, King's College London, UK; Fern Day, PhD, Department of Psychosis Studies, Biomedical Research Centre, Institute of Psychiatry, Psychology and Neuroscience, King's College London, UK; Lucia Valmaggia, PhD, Department of Psychosis Studies, Biomedical Research Centre, Institute of Psychiatry, Psychology and Neuroscience, King's College London, UK; Grazia Rutigliano, MD, Department of Psychosis Studies, Biomedical Research Centre, Institute of Psychiatry, Psychology and Neuroscience, King's College London, UK, and Department of Clinical and Experimental Medicine, University of Pisa, Pisa, Italy; Andrea De Micheli, MD, Department of Psychosis Studies, Biomedical Research Centre, Institute of Psychiatry, Psychology and Neuroscience, King's College London, UK, and Department of Brain and Behavioural Sciences, University of Pavia, Pavia, Italy; Paolo Fusar-Poli, PhD, Department of Psychosis Studies, Biomedical Research Centre, Institute of Psychiatry, Psychology and Neuroscience, King's College London, UK; Philip McGuire, PhD, Department of Psychosis Studies, Biomedical Research Centre, Institute of Psychiatry, Psychology and Neuroscience, King's College London, UK

Correspondence: Arsime Demjaha, Department of Psychosis Studies, Biomedical Research Centre, Institute of Psychiatry, Psychology and Neuroscience, King's College London, 16 De Crespigny Park, London SE5 8AF, UK. E-mail: arsime.demjaha@kcl.ac.uk

First received 4 Dec 2016, final revision 12 Jun 2017, accepted 19 Jun 2017
}

\section{Funding}

This work was supported by the UK Medical Research Council and the National Institute for Health Research (NIHR) Mental Health Biomedical Research Centre at South London and Maudsley NHS Foundation Trust and King's College London. The views expressed are those of the author(s) and not necessarily those of the NHS, the NIHR or the Department of Health. The Outreach and Support in South London (OASIS) service was supported by the Guy's and St Thomas' Charitable Foundation, South London and Maudsley Trust.

\section{Acknowledgements}

We thank the members of the Outreach and Support in South London (OASIS) team who were involved in the recruitment, management and clinical follow-up of the participants reported in this manuscript. Our special thanks go to the service users of OASIS.

\section{References}

1 Bleuler E. Dementia Praecox or the Group of Schizophrenias. International University Press, 1950.

2 Demiaha A, Morgan K, Morgan $\mathrm{C}$, Landau S, Dean $\mathrm{K}$, Reichenberg A, et al. Combining dimensional and categorical representation of psychosis: the way forward for DSM-V and ICD-11? Psychol Med 2009; 39: 1943-55. 
3 Demjaha A, Valmaggia L, Stahl D, Byrne M, McGuire P. Disorganization/cognitive and negative symptom dimensions in the at-risk mental state predict subsequent transition to psychosis. Schizophr Bull 2012; 38: 351-9.

4 Harrow $\mathrm{M}$, Silverstein $\mathrm{M}$, Marengo J. Disordered thinking: does it identify nuclear schizophrenia? Arch Gen Psychiatry 1983; 40: 765-71.

5 Crow TJ. Nuclear schizophrenic symptoms as a window on the relationship between thought and speech. Br J PSychiatry 1998; 173: 303-9.

6 Asarnow RF. Neurocognitive impairments in schizophrenia: a piece of the epigenetic puzzle. Eur Child Adolesc Psychiatry 1999; 8 (suppl 1): 15-8.

7 Norman RM, Malla AK, Cortese L, Cheng S, Diaz K, McIntosh E, et al. Symptoms and cognition as predictors of community functioning: a prospective analysis Am J Psychiatry 1999; 156: 400-5.

8 McGuire PK, Quested DJ, Spence SA, Murray RM, Frith CD, Liddle PF. Pathophysiology of 'positive' thought disorder in schizophrenia. Br J Psychiatry 1998; 173: 231-5.

9 Palaniyappan L, Mahmood J, Balain V, Mougin O, Gowland PA, Liddle PF. Structural correlates of formal thought disorder in schizophrenia: an ultra-high field multivariate morphometry study. Schizophr Res 2015; 168: 305-12.

10 Kircher $T$, Liddle $P$, Brammer M, Murray R, McGuire P. Neural correlates of "negative" formal thought disorder. Der Nervenarzt 2003; 74: 748-54.

11 Bearden CE, Wu KN, Caplan R, Cannon TD. Thought disorder and communication deviance as predictors of outcome in youth at clinical high risk for psychosis. J Am Acad Child Adolesc Psychiatry 2011; 50: 669-80.

12 Phillips LJ, Yung AR, McGorry PD. Identification of young people at risk of psychosis: validation of Personal Assessment and Crisis Evaluation Clinic intake criteria. Aust N Z J Psychiatry 2000; 34 (suppl): S164-9.

13 Miller TJ, McGlashan TH, Woods SW, Stein K, Driesen N, Corcoran CM, et al. Symptom assessment in schizophrenic prodromal states. Psychiatr Q 1999; 70: 273-87.

14 Schultze-Lutter F, Michel C, Schmidt SJ, Schimmelmann BG, Maric NP Salokangas RK, et al. EPA guidance on the early detection of clinical high risk states of psychoses. Eur Psychiatry 2015; 30: 405-16.

15 Lo Cascio $N$, Saba $R$, Hauser $M$, Vernal $D L$, Al-Jadiri $A$, Borenstein $Y$, et al. Attenuated psychotic and basic symptom characteristics in adolescents with ultra-high risk criteria for psychosis, other non-psychotic psychiatric disorders and early-onset psychosis. Eur Child Adolesc Psychiatry 2016; 25: 1091-102.

16 Cornblatt BA, Carrion RE, Auther A, McLaughlin D, Olsen RH, John M, et al. Psychosis prevention: A modified clinical high risk perspective from the recognition and prevention (RAP) program. Am J Psychiatry 2015; 172: 986-94.

17 Addington J, Liu L, Buchy L, Cadenhead KS, Cannon TD, Cornblatt BA, et al. North American Prodrome Longitudinal Study (NAPLS 2): the prodromal symptoms. J Nerv Ment Dis 2015; 203: 328-35.

18 DeVylder JE, Muchomba FM, Gill KE, Ben-David S, Walder DJ, Malaspina D, et a symptom trajectories and psychosis onset in a clinical high-risk cohort: the relevance of subthreshold thought disorder. Schizophr Res 2014; 159: 278-83.

19 Hartmann JA, Yuen HP, McGorry PD, Yung AR, Lin A, Wood SJ, et al. Declining transition rates to psychotic disorder in "ultra-high risk" clients: investigation of a dilution effect. Schizophr Res 2016; 170: 130-6.

20 Gooding D, Ott S, Roberts S, Erlenmeyer-Kimling L. Thought disorder in mid-childhood as a predictor of adulthood diagnostic outcome: findings from the New York High-Risk Project. Psychol Med 2013; 43: 1003-12.

21 Liddle PF, Ngan ET, Caissie SL, Anderson CM, Bates AT, Quested DJ, et al. Thought and Language Index: an instrument for assessing thought and language in schizophrenia. Br J Psychiatry 2002; 181: 326-30.

22 Sommer IE, Derwort AM, Daalman K, de Weijer AD, Liddle PF, Boks MP. Formal thought disorder in non-clinical individuals with auditory verbal hallucinations. Schizophr Res 2010; 118: 140-5.

23 Fusar-Poli P, Byrne M, Badger S, Valmaggia LR, McGuire PK. Outreach and support in south London (OASIS), 2001-2011: ten years of early diagnosis and treatment for young individuals at high clinical risk for psychosis. Eur Psychiatry 2013; 28: 315-26.

24 Yung AR, Phillips $\amalg$, McGorry PD, McFarlane CA, Francey S, Harrigan S, et al. Prediction of psychosis. A step towards indicated prevention of schizophrenia. Br J Psychiatry Suppl 1998; 172: 14-20.

25 Huber G, Gross G, Schuttler R, Linz M. Longitudinal studies of schizophrenic patients. Schizophr Bull 1980; 6: 592-605.

26 Murray HA. Thematic Apperception Test. Harvard University Press, 1943.

27 American Psychiatric Association. Diagnostic and Statistical Manual of Mental Disorders (Fourth Edition, Text Revision) (DSM-IV-TR). American Psychiatric Association, 2000.

28 Nelson HE, O'Connell A. Dementia: the estimation of premorbid intelligence levels using the New Adult Reading Test. Cortex 1978; 14: 234-44.

29 Coren S. Measurement of handedness via self-report: the relationship between brief and extended inventories. Percept Mot Skills 1993; 76: 1035-42.

30 Fusar-Poli P, Rocchetti M, Sardella A, Avila A, Brandizzi M, Caverzasi E, et al. Disorder, not just state of risk: meta-analysis of functioning and quality of life in people at high risk of psychosis. Br J Psychiatry 2015; 207: 198-206.

31 Schultze-Lutter F, Addington J, Ruhrmann S, Klosterkötter J. Schizophrenia Proneness Instrument, Adult Version (SPI-A). Giovanni Fioriti, 2007.

32 Ott SL, Roberts S, Rock D, Allen J, Erlenmeyer-Kimling L. Positive and negative thought disorder and psychopathology in childhood among subjects with adulthood schizophrenia. Schizophr Res 2002; 58: 231-9.

33 Armando M, Pontillo M, De Crescenzo F, Mazzone L, Monducci E, Lo Cascio N, et al. Twelve-month psychosis-predictive value of the ultra-high risk criteria in children and adolescents. Schizophr Res 2015; 169: 186-92.

34 Caplan R. Thought disorder in childhood. J Am Acad Child Adolesc Psychiatry 1994; 33: 605-15.

35 Valmaggia LR, Stahl D, Yung AR, Nelson B, Fusar-Poli P, McGorry PD, et al. Negative psychotic symptoms and impaired role functioning predict transition outcomes in the at-risk mental state: a latent class cluster analysis study. Psychol Med 2013; 43: 2311-25. 